\section{Home}

Copyright

Welcome Message from

General Co-Chairs

Message from TPC Co-

Chairs

Message from Dissertation Digest Chairs

Committees

Program-At-A-Glance

Distinguished Experts Panel

Panels

Keynote Speeches

Tutorials

Table of Contents

Workshops

Author Index

Patrons

Search

Help

\footnotetext{
$\underline{\text { Technical support } \mathcal{E}}$ inquiries

Research Publishing Services

$t:+65-64921137$

$f:+65-67474355$

e:enquiries@rpsonline.com.sg
}

\section{Proceedings of the \\ 2012 IEEE Network Operations and Management Symposium}

\author{
16 - 20 April 2012 \\ Maui, Hawaii, USA
}

Editors:

Filip De Turck, Ghent University-IBBT, Belgium

Luciano Paschoal Gaspary, UFRGS, Brazil

Deep Medhi, University of Missouri-Kansas City, USA

IEEE Catalog Number: CFP12NOM-USB

ISBN: 978-1-4673-0268-5

\section{Organized By

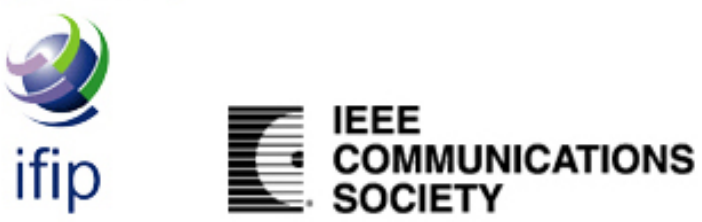

Copyright and Reprint Permission: Abstracting is permitted with credit to the source. Libraries are permitted to photocopy beyond the limit of U.S. copyright law for private use of patrons those articles in this volume that carry a code at the bottom of the first page, provided the per-copy fee indicated in the code is paid through Copyright Clearance Center, 222 Rosewood Drive, Danvers, MA 01923. For other copying, reprint or republication permission, write to IEEE Copyrights Manager, IEEE Operations Center, 445 Hoes Lane, Piscataway, NJ 08854. All rights reserved. Copyright @ 2012 by IEEE. 
file:///C|/Users/dstevens/Documents/_D_Drive/dstevens/Publicaties\%20I NTEC/5219_i_i.htm|[6/28/2012 11:36:05 AM]

IEEE/IFIP NOMS 2012 11:36:05 AM]

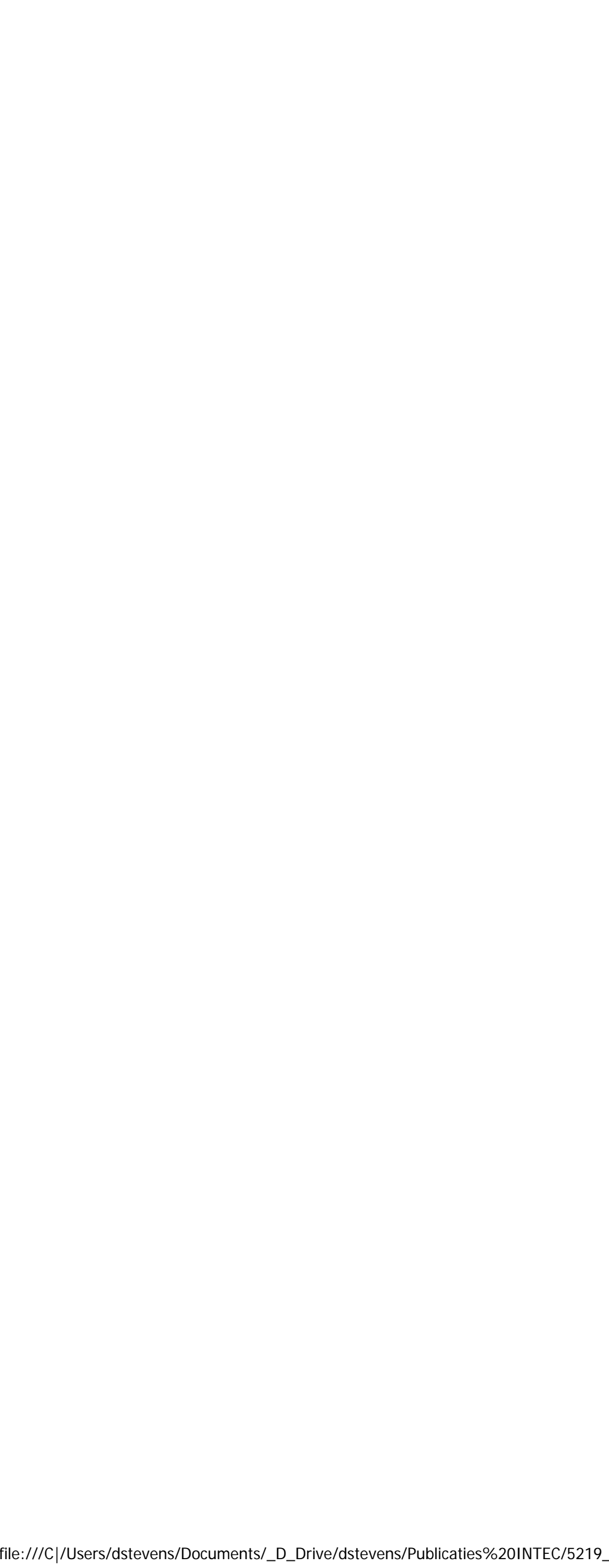

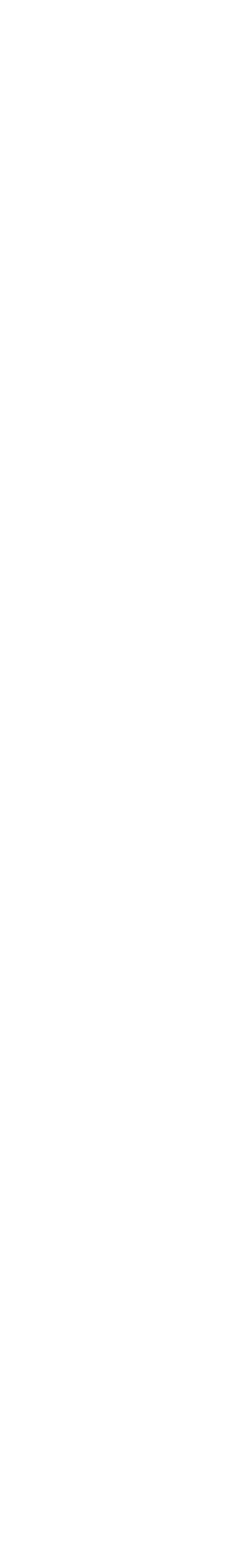




\title{
SeCoA: Autonomous Semantic Service Composition Algorithm in Symbiotic Networks
}

\author{
Tim De Pauw* ${ }^{* \dagger}$, Bruno Volckaert*, Veerle Ongenae ${ }^{\dagger}$ and Filip De Turck* \\ ${ }^{*}$ Department of Information Technology (INTEC) \\ Ghent University - IBBT, Gaston Crommenlaan 8 bus 201, 9050 Ghent, Belgium \\ ${ }^{\dagger}$ Faculty of Applied Engineering Sciences (INWE) \\ University College Ghent, Schoonmeersstraat 52, 9000 Ghent, Belgium \\ Email: tim.depauw@intec.ugent.be
}

\begin{abstract}
We propose symbiotic networks, a novel approach toward sharing of network resources in order to increase the scalability, dependability and energy efficiency of colocated networks. As symbiotic networks offer large amounts of software services, one challenge is to allow these services to operate "symbiotically" as well. By combining services from different parties, service compositions arise, which allow for a richer set of functionality. Creating such compositions, however, requires intricate knowledge about services and their interoperability. Using a semantic domain and service model, we describe $\mathrm{SeCoA}$, a tunable best-first search algorithm for autonomously constructing symbiotic service compositions. A performance evaluation of SeCoA was conducted, showing that the algorithm offers acceptable performance for moderately sized compositions.
\end{abstract}

\section{INTRODUCTION}

Over the past few years, home as well as office environments have seen a dramatic increase in the amount of coexistent networks, both wired and wireless. Aside from public cellular networks, many premises nowadays are equipped with several proprietary Ethernet networks as well as, for instance, home automation networks. 3G, Wi-Fi, ZigBee, and so forth are all contending for the same limited amount of bandwidth. Thus, the ever-growing density of both communication networks and devices is becoming a serious predicament.

Through the introduction of so-called symbiotic networks, our goal is to mitigate this phenomenon. We aim to allow networks to communicate transparently across logical and physical boundaries, by sharing media and services in an intelligent manner, such that they can operate as if they were symbiotic organisms. The symbiotic networks we envisage are highly cooperative and autonomously managed. By handling resources more prudently, we hope to make strides in the fields of scalability, dependability and energy efficiency.

In this paper, we focus on the sharing of software services in such symbiotic networks. Usually, individual networks expose certain functionality through a set of services. By establishing symbiosis, we will allow for them to automatically discover and call upon each other's services. Thus, we aspire to render the services as symbiotic as their environments. This will allow for novel cooperation opportunities, resulting in service infrastructures much greater than the sum of their parts.

978-1-4673-0269-2/12/\$31.00 (c) 2012 IEEE
As an initial step toward full symbiosis, we introduce SeCoA, a best-first search algorithm for the semantic composition of services. Using a taxonomy of services, we mix and match them in order to ultimately attain a certain objective. In a dynamic environment where each network offers hundreds of services, the task of aggregating them into rich compositions poses a vastly complex problem, unsuitable for manual intervention. Therefore, we wish to provide a service platform capable of autonomous discovery and composition of services.

\section{RELATED WORK}

Many researchers have tackled service composition, semantic or otherwise. In [1], the $O W L-S$ ontology is compared to other formalisms used in this field, such as BPEL. Notably, the authors mention that only OWL-S allows for expressing nonfunctional properties such as Quality of Service; we expect these to be of vital importance in symbiotic networks. While OWL-S is mostly incomplete in this area, OWL provides the underpinnings for a highly expressive model.

The semi-automatic OWL-S service composer by Sirin et al. [2] guides the user in mostly manual composition creation. The hybrid $O W L S-M X$ Matchmaker [3] extends this principle by attempting to select the most suitable service, using logicbased reasoning and text similarity measurement.

OWLS-XPlan [4] combines a fast-forward planner with an HTN planner; its intermediate translation to PDDL could raise performance concerns in resource-constrained environments such as symbiotic networks. Similarly, SHOP2 [5] relies on HTN to build OWL-S service compositions, using a proprietary model. One can also translate OWL-S to BPEL. [6] In the WTE+ project [7], [8], dynamic composition of Web Services was achieved through QoS-aware HTN planning, focused on building mashups and reducing development times.

SeCoA differs from such previous efforts in that it takes into account additional, non-functional constraints, related to the symbiotic nature of the network environments involved. Additionally, a tunable priority function is introduced.

At this point, we assume policies to be statically defined. Bahati et al. [9] analyze the modifications policies may undergo and implement an adaptive approach. Conflict resolution in ontology-based policies is discussed by Barron et al. [10] 
In terms of performance evaluation, a few ontologies have been used for benchmarking. The University Ontology Benchmark [11] provides ontologies of varying sizes to assess a system's inference capabilities. Specifically for OWL-S, the OWL-S Service Retrieval Test Collection (OWLS-TC) [12] can be used to evaluate composition algorithms. However, since our approach currently relies on a subset of OWL-S, we developed a random ontology generator tailored to it.

\section{Assumptions}

Before we can outline our approach toward semantic service composition, it is necessary to describe the general model we maintain throughout this paper.

Similar to [4] and [7], we assume that each service has a list of input and output parameters. While both may be empty, composition is parameter-based: given an initial service, a goal service, and a set of other available services, the objective is to create a directed acyclic graph $G=(V, E)$ such that

1) each element of $V$ corresponds to a service,

2) each element of $E$ represents the exchange of a parameter value between two services,

3) there is at least one path from the vertex for the initial service to that for the goal service, and

4) the value of each input parameter of each service included in the composition is provided by that of exactly one output parameter of another service.

Each parameter has a type, given by a class name. Classes may optionally be equivalent or inherit from one another. If service $A$ produces an output parameter $x$ of type $X$, and service $B$ requires an input parameter $y$ of type $Y$, then the value of $x$ may be used as the value of $y$ if and only if $X$ is either equivalent to $Y$ or a subclass of it.

An important aspect of symbiotic networks is that services in a composition may belong to different parties, who agree upon a common vocabulary. To govern the interoperation of these parties' services, we introduce so-called policies. A policy is of the form antecedent $\Rightarrow$ consequent. If the condition expressed by the antecedent is met by an edge $e \in E$, then the consequent is applied to the services represented by the vertices which $e$ connects.

An antecedent checks if the input parameter, output parameter, and the services respectively providing and consuming them meet certain requirements. The most trivial of antecedents states that the providing service is part of some administrative domain, and the consuming service of another. We assume antecedents to be freeform rules, which apply to properties of the edge $e$, the service vertices which it connects, and the environment in which they exist.

The application of a consequent consists of the introduction of up to two filters. An output filter may be applied to the output parameter that is being provided, and an input filter to the input parameter consumed. These filters are an abstract representation of non-functional requirements, which we are aiming to extend in the future. Examples include a filter stating that the parameter value must be transmitted securely, or that it must be measured with a certain accuracy.
TABLE I

OWL OBJECT PROPERTIES

\begin{tabular}{ccc} 
Domain & Property & Range \\
\hline Policy & hasAntecedent & Antecedent \\
Policy & hasConsequent & Consequent \\
Consequent & appliesOutputFilter & Filter \\
Consequent & appliesInputFilter & Filter \\
service:Service & belongsTo & AdministrativeDomain
\end{tabular}

\section{APPROACH}

\section{A. Ontology Extensions}

The OWL-S standard provides the foundations to semantically describe and operate services, but naturally, it does not explicitly provide a framework to do so in symbiotic networks. Therefore, in this section, we introduce an initial set of extensions to the ontology, which allow us to express symbiotic-network-specific concepts.

Our assumptions from Section III yield the OWL classes Policy, Antecedent, Consequent, and Filter; the associated OWL properties are shown in Table I. An Antecedent is a SWRL Expression using the variables PolicyStartService, PolicyEndService, PolicyOutput, and Policylnput. We also introduce the class PolicyList to impose an order relation upon policies. Finally, we use the class AdministrativeDomain to link services to the symbiotic networks which provide them, so that we can use this information in policy antecedents.

As an example, consider Wireless Body Area Networks (WBAN), which place sensor equipment on or near the human body, usually for the purpose of medical monitoring. In our scenario, a sensor exposes the patient's body temperature in degrees Fahrenheit, while the hospital's equipment only understands the Celsius scale. However, the hospital network conveniently provides a conversion service as well. In addition, to ensure the confidential treatment of medical information, a policy is instituted. It requires encryption of all data transmitted from the administrative domain encompassing the patient's WBAN to the hospital's administrative domain:

\section{belongsTo(PolicyStartService, Patient) \\ $\wedge$ belongsTo(PolicyEndService, Hospital) \\ $\Rightarrow$ apply EncryptionFilter to PolicyOutput and apply DecryptionFilter to PolicyInput}

Figure 1 visualizes the eventual composition incorporating the services and applying the policy. For the sake of simplicity, the services' OWL-S processes are not explicitly shown.

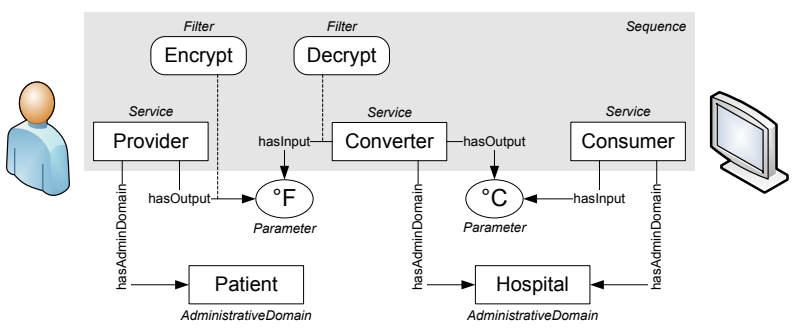

Fig. 1. Example composition ontology (simplified) 


\section{B. Composition Methodology}

Having described our ontology model, we can now present our three-phase service composition algorithm SeCoA.

1) Phase 1: Weed Out Unsatisfiable Inputs: In the first phase, SeCoA eliminates services which require input parameters that are not provided by any of their counterparts, as these can never take part in a composition. If neither the type of an input, nor a subtype of it can be provided, the service requiring that particular type will not be considered any further.

2) Phase 2: Match Outputs to Inputs: The best-first search algorithm for constructing a composition is shown in Figure 2; it was partly inspired by the WTE+ algorithm [7]. Starting from the goal service, it attempts to work its way up to the initial service by finding services that provide unsatisfied input parameters. Thus, initially, the solution graph only contains the goal service, and its input parameters are to be found.

In the first iteration, one of these unknown inputs is examined. For each service which provides an output parameter of the same class or a subclass, a queue entry is created.

Subsequent iterations gradually satisfy the remaining inputs until the first valid solution is encountered. If a service is already part of the composition, the corresponding graph vertex is reused. Additional checks are performed to make sure that the resulting composition does not contain any cycles; this is exemplified by the call to hasAncestor().

The order in which subsequent incomplete compositions are selected for expansion depends on their priority, given by $p(c)=\alpha \times u_{c}+\beta \times s_{c}+\gamma \times d_{c}$, where $c$ is the partial composition being examined, $u_{c}$ is the number of inputs in $c$ which are still unsatisfied, $s_{c}$ is the number of services involved in $c, d_{c}$ is the number of administrative domains involved in $c$, and $\alpha, \beta$ and $\gamma$ are tunable weight coefficients.

3) Phase 3: Apply Policies: Provided that phase 2 produced a valid composition, the list of policies is exhaustively checked against each of its parameter exchanges. If an antecedent match is encountered, the output and/or input filter associated with the policy's consequent are applied to the parameters.

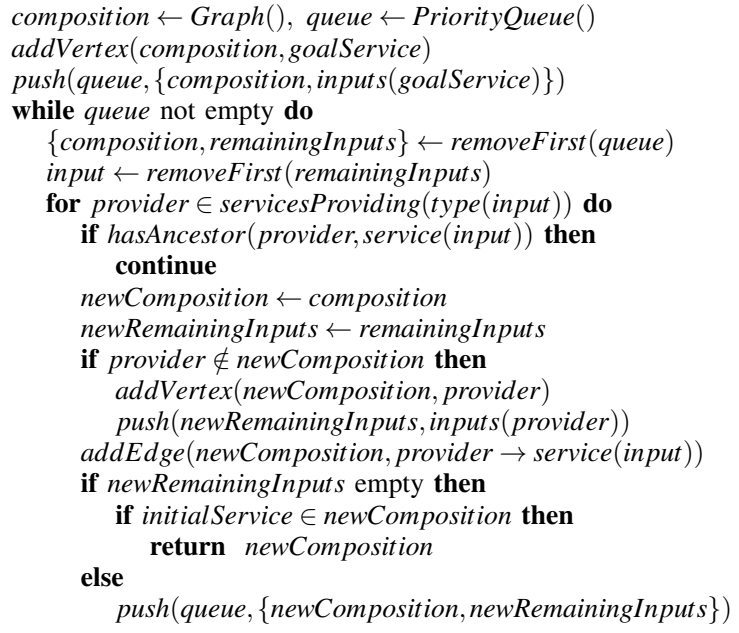

Fig. 2. SeCoA phase 2: Match outputs to inputs

\section{Performance Evaluation Results}

The composition algorithm described in the previous section was implemented in Java 6, using version 1.1 .0 beta of the $O W L-S$ API [13] and with Pellet [14] as a reasoner.

\section{A. Evaluation Methodology}

To benchmark the algorithm's performance, we applied it to randomly generated service and policy ontologies. The following approach was used to construct these:

- First, a set of OWL classes is created, representing parameter types. Each class has a configurable probability of being either equivalent to or being a subclass of another.

- Next, the services are generated. Each of them is assigned a set of in- and output parameters, the size of which is randomly selected with a uniform distribution.

- Subsequently, a set of AdministrativeDomain individuals is created and the services are randomly assigned to them.

- Finally, for each pair of administrative domains, a policy, an input filter, and an output filter are generated. The policy's antecedent states that the providing service must belong to the first administrative domain and the consuming one to the second; its consequent applies the filters to the two parameters involved.

SeCoA was applied to the generated ontologies on the WiLab testbed [15]. While primarily a sensor network testbed, WiLab's Linux machines with a $500 \mathrm{MHz}$ processor and $256 \mathrm{MB}$ of RAM are similar to many devices installed in potential symbiotic networks and provide interesting insights into resource-constrained environments. In previous work, we showed that the machines are capable of performing common ontological reasoning tasks. [16] WiLab's large number allowed us to run simulations in parallel.

The problem generator's parameters and their values are shown in Table II; we used three different configurations, differing in the number of parameter types and services, and generated 100 problems using each of them. We chose $\alpha, \beta$ and $\gamma$ such that the number of unresolved inputs would always take precedence over the number of services and domains.

\section{B. Evaluation Results}

We found SeCoA to be able of tackling most of the generated problems. For the three configurations shown in Table II, respectively 88,87 and 85 problems yielded a solution after an average of 275 iterations of phase 2 .

Figure 3 provides insight into the generated compositions. Predictably, as the problem scale rises, so do all three metrics.

TABLE II

Problem Generator Parameters

\begin{tabular}{ccc} 
Parameter & Description & Value(s) \\
\hline$\# T$ & parameter types & $\{10,15,20\}$ \\
$P_{E}$ & probability of type equivalence & $10 \%$ \\
$P_{I}$ & probability of type inheritance & $10 \%$ \\
$\# S$ & services (without initial and goal) & $\{50,75,100\}$ \\
{$\left[\# I_{m}, \# I_{M}\right]$} & input parameters per service & {$[1,5]$} \\
{$\left[\# O_{m}, \# O_{M}\right]$} & output parameters per service & {$[1,5]$} \\
$\# D$ & administrative domains & 3
\end{tabular}


The execution times of our algorithm's three phases, as well as for loading the required ontologies and reason upon them, are shown in Figure 4. Here too, problem complexity appears to have the same impact on every phase of the algorithm.

The execution time for phase 2 is nearly entirely defined by the time to identify services providing a certain output type. After all, this phase involves reasoning on the type ontology. Phase 3, policy processing, is the most time-consuming one overall. Matching nine SWRL rules against every edge in the composition turns out to be quite the strain on resources.

The accumulated execution times may seem rather high, but in symbiotic networks where the service model does not change too frequently, we expect these to be acceptable. Moreover, for the scenarios considered here, we feel our testbed's limited resources provided us with adequate performance.

Contrary to what the charts might suggest, there was no immediate correlation between the number of services or parameter exchanges on one hand, and the algorithm's total execution time on the other. The reason for this lies in the fact that ontological reasoning is the prominent factor in phases 2 and 3. Its requirements depend on the ontology's complexity, for which no truly dependable measure exists.

Finally, a brief word on memory usage. While reasoning is traditionally a memory-intensive process, WiLab's modest hardware coped with SeCoA rather well. We noted average memory footprints of $63,890 \mathrm{~KB}, 65,215 \mathrm{~KB}$ and $72,933 \mathrm{~KB}$ for our three configurations, and a maximum of $134,716 \mathrm{~KB}$.

\section{CONCLUSiOnS AND Future WORK}

We introduced the concept of symbiotic networks as a means to enable intensive collaboration and resource sharing in the home and office networks of the future. To allow services in such networks to cooperate in an equally symbiotic fashion, we described SeCoA, a tunable best-first search algorithm for constructing cross-network, cross-layer service compositions. Using semantic reasoning as the foundation of the algorithm, we are able to create rich representations of services and use them in the composition process. Through extensive experiments, we showed that SeCoA offers acceptable performance.

The presented algorithm will serve as a basis for future research. First off, we aim for full IOPE support rather than parameter matching only. We are also planning more extensive support of OWL and OWL-S, to harvest the full potential of semantic service representation. In addition, the SWRL-based policy mechanism will be enhanced with more expressiveness. Since policy matching is currently rather time-consuming, we will study the performance improvements induced by distributing the reasoning process. Finally, the compositions resulting from $\mathrm{SeCoA}$ will be applied to real-life scenarios.

\section{ACKNOWLEDGMENT}

Tim De Pauw would like to thank the University College Ghent Research Fund for financial support through his Ph.D. grant. Part of this work has been funded by the IWT SBO SymbioNets project. We are grateful to our colleague Anna Hristoskova for the interesting discussions on service composition.

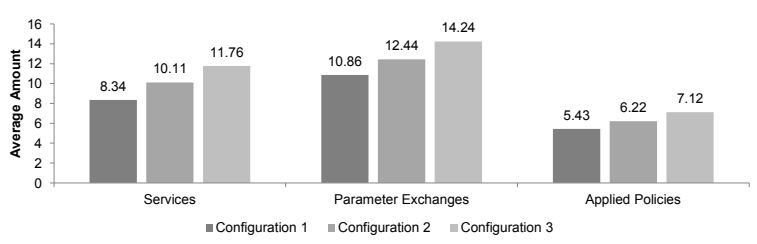

Fig. 3. Composition metrics for problems generated according to Table II

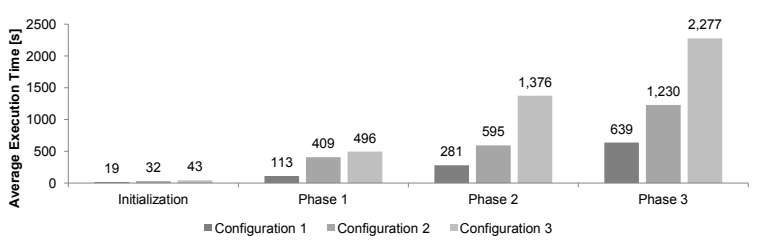

Fig. 4. Average execution times for problems generated according to Table II

\section{REFERENCES}

[1] N. Milanovic and M. Malek, "Current solutions for Web Service composition," IEEE Internet Comput., vol. 8, no. 6, pp. 51-59, 2004.

[2] E. Sirin, B. Parsia, and J. Hendler, "Filtering and selecting Semantic Web Services with interactive composition techniques," IEEE Intelligent Systems, pp. 42-49, 2004.

[3] M. Klusch, B. Fries, and K. Sycara, "Automated Semantic Web Service discovery with OWLS-MX," in Proc. 5th International Joint Conference on Autonomous Agents and Multiagent Systems (ACM AAMAS-06), Hakodate, Japan, 2006, pp. 915-922.

[4] M. Klusch, A. Gerber, and M. Schmidt, "Semantic Web Service composition planning with OWLS-XPlan," in Proc. AAAI Fall Symposium on Semantic Web and Agents, Arlington, VA, USA, 2005.

[5] E. Sirin, B. Parsia, D. Wu, J. Hendler, and D. Nau, "HTN planning for Web Service composition using SHOP2," Web Semantics: Science, Services and Agents on the WWW, vol. 1, no. 4, pp. 377-396, 2004.

[6] B. Bordbar, G. Howells, M. Evans, and A. Staikopoulos, "Model transformation from OWL-S to BPEL via SiTra," in Model Driven Architecture: Foundations and Applications. Springer, 2007, pp. 43-58.

[7] A. Hristoskova, B. Volckaert, and F. De Turck, "The WTE+ framework: automated construction and runtime adaptation of service mashups," Automated Software Engineering, pp. 1-44, 2012.

[8] - "Dynamic composition of semantically annotated web services through QoS-aware HTN planning algorithms," in Proc. 4th IEEE International Conference on Internet and Web Applications and Services (ICIW 2009), 2009, pp. 377-382.

[9] R. Bahati and M. Bauer, "Towards adaptive policy-based management," in Proc. 12th IEEE/IFIP Network Operations and Management Symposium (NOMS 2010), Osaka, Japan, 2010.

[10] J. Barron, S. Davy, and B. Jennings, "Conflict analysis during authoring of management policies for federations," in Proc. 1st IFIP/IEEE Workshop on Managing Federations and Cooperative Management (ManFed.CoM 2011), Dublin, Ireland, 2011.

[11] L. Ma, Y. Yang, Z. Qiu, G. Xie, Y. Pan, and S. Liu, "Towards a complete OWL ontology benchmark," The Semantic Web: Research and Applications, vol. 4011, pp. 125-139, 2006.

[12] M. Klusch, P. Kapahnke, B. Fries, M. A. Khalid, and M. Vasileski. (2010) OWL-S Service Retrieval Test Collection. [Online]. Available: http://semwebcentral.org/projects/owls-tc/

[13] OWL-S API. [Online]. Available: http://mindswap.org/2004/owl-s/api

[14] E. Sirin, B. Parsia, B. Grau, A. Kalyanpur, and Y. Katz, "Pellet: A practical OWL-DL reasoner," Web Semantics: Science, Services and Agents on the World Wide Web, vol. 5, no. 2, pp. 51-53, Jun. 2007.

[15] L. Tytgat, B. Jooris, P. De Mil, B. Latré, I. Moerman, and P. Demeester, "Demo abstract: WiLab, a real-life wireless sensor testbed with environment emulation," in Proc. 6th European conference on Wireless Sensor Networks (EWSN 2009), Cork, Ireland, 2009.

[16] T. De Pauw, B. Volckaert, F. De Turck, and V. Ongenae, "On the design of a flexible software platform for in-building OTT service provisioning," in Proc. 4th IFIP/IEEE Workshop on Distributed Autonomous Network Management Systems (DANMS 2011), Dublin, Ireland, 2011. 